\title{
Comparing the effect of two different exercise types, mini- trampoline and fast-walking to gastrocnemius/soleus muscle elasticity by sonoelastrography
}

\author{
Tuba Tülay Koca ${ }^{1} \odot$, Murat Baykara ${ }^{2} \oplus$, Adnan Demirel $^{1} \odot$, Ejder Berk $^{1} \odot$ \\ ${ }^{I}$ Department of Physical Medicine and Rehabilitation, Sütçü Imam University School of Medicine, Kahramanmaraş, Turkey \\ ${ }^{2}$ Department of Radiology, Sütçü Imam University School of Medicine, Kahramanmaraş, Turkey
}

DOI: $10.18621 /$ eurj.407527

\begin{abstract}
Objectives: Sonoelastography is a newly introduced ultrasound technique that evaluates tissue elasticity and thus provides additional information to that offered by conventional ultrasound images. Here, we compared the effect of ballistic jumping on mini-trampolin with walk exercise to the elasticity of gastrocnemius/soleus muscles with quantitative measurements by sonoelastography.

Methods: Forty volunteer healty male cases between the ages of 20-22 years were included the study. Initially, both exercise groups were subjected to joint warm-up movements for 8 minutes. Twenty of the cases (40 legs) had mini-trampoline (balistic jumping) exercise for 15 minutes. Another 20 cases (40 legs) had 15 minutes fast-walk exercise. Measurements were made immediately before and after the procedure (within 5 minutes) with the sonoelastography.

Results: A statistically significant difference was observed in the gastrocnemius/soleus muscles after exercise in the trampoline group according to sonoelastography strain value (SESV) data separately $(p=0.0001 / p=$ 0.0001). According to this, when the SESV values evaluated after 15 minutes of jump with the trampoline, we obtained that the elasticity increases and stifness decreases in the calf muscles. In the walking group, no statistically difference was observed in the gastrocnemius/soleus muscles after exercise separately $(p=0.7925$ $/ p=0.1879$ ).

Conclusions: According to the study, in the trampoline group in general, a decrease in strain values and an increase in elasticityin muscle groups were found; in the walking group, a decrease in muscle elasticity, an increase in stiffness and muscle strain were found eventually. We found that the 15 -minutes exercise program on the mini-trampoline is more effective as a heating technique and has more positive effect on muscle elasticity than the 15-minutes walking exercise.
\end{abstract}

Keywords: sonoelastography, trampoline, walk, exercise, muscle, stifness, strain

Received: March 19, 2018; Accepted: July 5, 2018; Published Online: November 12, 2018

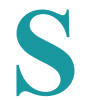
onoelastography (SE) is a newly introduced ultrasound technique that evaluates tissue elasticity and thus provides additional information to that offered by conventional ultrasound images [1]. It is a noninvasive method for measuring tissue elasticity whereby a quantitative estimate is obtained of the elasticity of various soft tissues, including muscles, tendons, salivary glands, and abdominal organs. The

Address for correspondence: Tuba Tülay Koca, MD., Assistant Professor, Sütçü Imam University School of Medicine, Department of Physical Medicine and Rehabilitation, Kahramanmaraş,Turkey

E-mail: tuba_baglan@yahoo.com 
imaging method is based on acoustic radiation force impulses through the tissue to obtain an elastic modulus. The result is a local measurement of the tissue elasticity at each point of interest of an organ. This imaging method is operator independent, reproducible, and quantitative [2-4].

It has been documented that exercises on a minitrampoline involve a multi-component approach, including muscle coordination, strength and balance training, body stability, and joint flexibility training [5]. Muscle action and coordination in the lower extremities were continuously facilitated by maintaining body balance on an elastic surface. In general, the ability to maintain balance is based on three mechanisms, including increasing the base of support, counter-rotating segments around the center of mass, and applying an external force other than the ground reaction force. In performing the mini-trampoline exercise, the participants were challenged to stabilize their body while keeping the center of mass over the base of the support. They needed to exert muscle force and neuromuscular responses to stiffen their legs in order to overcome the unstable conditions [6].

The effect of different exercise types on muscle elasticity has not been studied sufficiently. To best of our knowledge, there is no study about mini-trampolin exercise's acute effect to gastroknemius/soleus muscle' elasticity, and the comparison of the results with fastwalk exercise by SE. Here, we compared the effect of mini-trampolin exercise with fast-walk exercise to the gastrocnemius/soleus muscles with quantitative measurements by SE.

\section{METHODS}

\section{Study Population}

Forty volunteer healty male cases between the ages of 20-22 years were included the study. Initially, both exercise groups were subjected to joint warm-up movements for 8 minutes. Twenty of the cases (40 legs) had mini-trampoline (balistic jumping) exercise for 15 minutes. Another 20 cases (40 legs) had 15 minutes fast-walk exercise. Measurements were made immediately before and after the procedure (within 5 minutes) with the SE.

Sonoelastography
SE is an ultrasound-based technique that determines mechanical properties of the tissue quantitatively, visually, and qualitatively compared with the B-mode analysis (acoustic impedance) or the color Doppler ultrasound (vascular blood flow). The diagnostic difficulties can be overcome by using the SE method, which is increasingly used in the recent years. Strain elastography is the most commonly used measurement technique among several others, and the externally applied pressure causes more deformation in the soft tissues and less deformation in the hard tissues. This information is color-coded on a screen, and the quantitative data are obtained by measurements from the region of interest (ROI) [2-4].

A high-resolution ultrasonography system (Aplio TM 400 Platinum, Toshiba Medical Systems Corporation, Tochigi, Japan) and a broad band linear probe (PLT-704SBT) were used. When the gastrocnemius / soleus muscles were visualized on a gray scale ultrasonography image of the area symmetrically, bilaterally and separately, elastography was inserted into the apparatus and pressure was applied. The SE strain value (SESV) of the subcutaneous fat tissue in the same image and SESV of the central segments of the muscles were detected using by measurements from ROI. The sonoelastography strain ratio (SESR) of the muscles was calculated by dividing the fat values by the muscle values. All procedures were performed by the same experienced radiologist who was kept uninformed about the clinical diagnosis.

\section{Statistical Analysis}

The approval for this study was granted by the Institutional Ethics Committee. Measurements and calculations derived from SE were expressed as mean \pm standard deviation. Categorical data were presented as numbers and percentages. The Shapiro-Wilk test was used to examine the fitness of the variables for the normal distribution. Comparisons of the obtained strain rates were made by independent Student's $t$ test. When $\mathrm{P}$ value was less than 0.05 , the result was considered statistically significant.

\section{RESULTS}

Forty male cases (80 legs) between 20-22 years of 
Table 1. Descriptive data of the groups

\begin{tabular}{lcccc}
\hline Group & & Age (year) & Height $(\mathbf{c m})$ & Weight $(\mathbf{k g})$ \\
\hline Trampoline & Mean \pm SD & $21 \pm 1.02$ & $174.5 \pm 6.13$ & $70 \pm 9.19$ \\
Walking & Mean \pm SD & $20.56 \pm 1.8$ & $176.5 \pm 4.4$ & $68.38 \pm 8.45$ \\
$\mathrm{t}$ & & 1.34 & 1.67 & 0.82 \\
$p$ value & & 0.18 & 0.09 & 0.41 \\
\hline SD $=$ standard deviation & &
\end{tabular}

age were included the study. There was no significant difference in age, height and weight between trampoline and walking groups $(p=0.182 / p=0.097$ / $p=0.414)$. The descriptive data for the groups are summarized in Table 1.

A statistically significant difference was observed in the gastrocnemius / soleus muscles after exercise in the trampoline group according to SESV data $(p=$
$0.0001 / p=0.0001)$ (Table 2). According to this, when the SESV values evaluated after 15 minutes of jump with the trampoline, we obtained that the elasticity increases and stiffness decreases in the calf muscles.

In the walking group, no statistically significant difference was observed in the gastrocnemius/soleus muscles after exercise $(p=0.7925 / p=0.1879)$ (Table $3)$. According to this, 15 minute fast-walking exercise

Table 2. Pre/post data of SESV values of gastrocnemius/soleus muscles in trampoline group

\begin{tabular}{lcc}
\hline Trampoline & Mean \pm SD & p value \\
\hline Gastroknemius First & $0.38 \pm 0.23$ & $\mathbf{0 . 0 0 0 1}$ \\
Gastroknemius Second & $0.15 \pm 0.08$ & \\
Soleus First & $0.59 \pm 0.32$ & $\mathbf{0 . 0 0 0 1}$ \\
Soleus Second & $0.22 \pm 0.08$ & \\
\hline SESV = sonoelastography strain value, SD &
\end{tabular}

$\mathrm{SESV}=$ sonoelastography strain value, $\mathrm{SD}=$ standard deviation

Table 3. Pre/post data of UESV values of gastrocnemius/soleus muscles in walking group

\begin{tabular}{lcc}
\hline Walking & Mean \pm SD & p value \\
\hline Gastroknemius First & $0.55 \pm 0.38$ & 0.7925 \\
Gastrocnemius Second & $0.52 \pm 0.61$ & \\
Soleus First & $0.72 \pm 0.58$ & 0.1879 \\
Soleus Second & $1.05 \pm 1.46$ &
\end{tabular}

$\mathrm{UESV}=$ ultrasound elastography strain value, $\mathrm{SD}=$ standard deviation

Table 4. Changes in SESR values before/after exercise in the gastrocnemius/soleus muscles

\begin{tabular}{llcc}
\hline Group & & $\begin{array}{c}\text { Gastrocnemius Percent } \\
(\mathbf{n = 4 0 )}\end{array}$ & $\begin{array}{c}\text { Soleus Percent } \\
(\mathbf{n}=\mathbf{4 0})\end{array}$ \\
\hline Trampoline & Mean \pm SD & $45.05 \pm 26.79$ & $46.60 \pm 24.29$ \\
& Median & 39.69 & 43.93 \\
& Minimum & 8.57 & 17.48 \\
Walking & Maximum & 80.00 & 86.11 \\
& Mean \pm SD & $161.80 \pm 251.74$ & $188.90 \pm 270.66$ \\
& Median & 59.38 & 84.57 \\
& Minimum & 3.23 & 3.57 \\
t & Maximum & 962.50 & 935.85 \\
$p$ value & & 2.9167 & 3.3118 \\
\hline
\end{tabular}

$\mathrm{SESR}=$ sonoelastography strain ratio, $\mathrm{SD}=$ standard deviation 

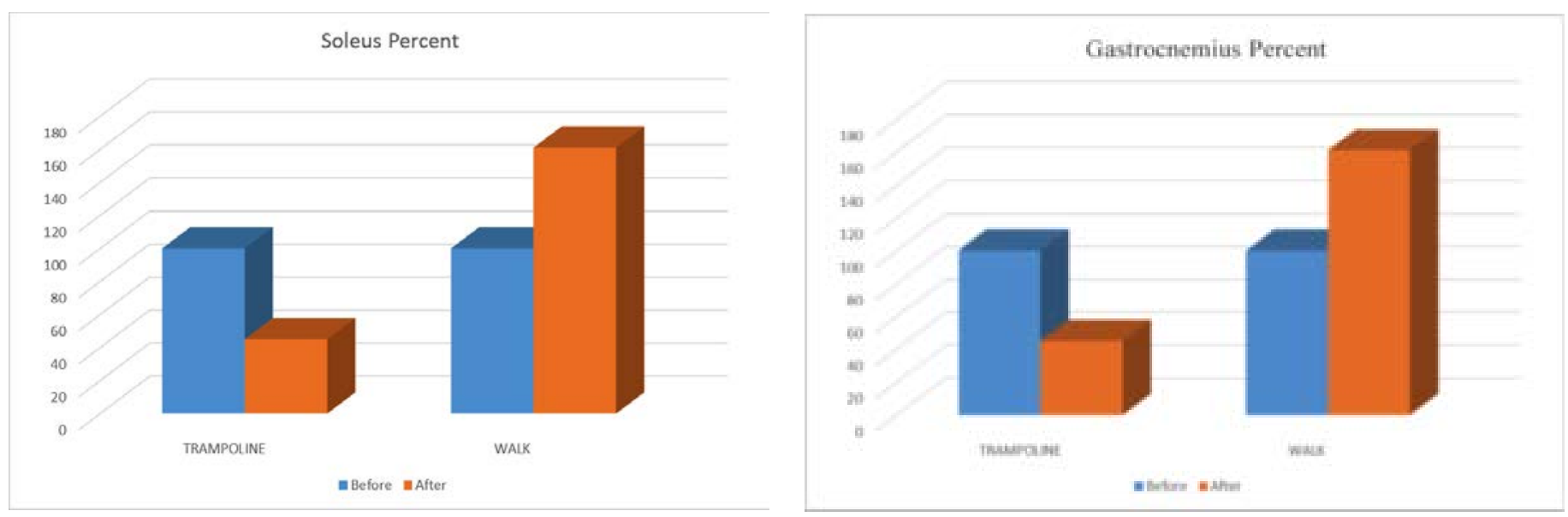

Figure 1. Graphical presentation of percent changes in SESRs in gastrocnemius/soleus muscles in trampoline and walk groups

did not cause any significant change in SESV valuesand did not affect muscle elasticity / stiffness positively.

When the percentages of SESR values before and after exercise were examined; the percent change in the both gastrocnemius/soleus muscle groups was significantly different at the trampoline group ( $p=$ $0.0046 / p=0.0014$ ) (Table 4). According to this, in the trampoline group in general, a decrease in strainvalues, an increase in elasticityatcalf muscles were found; in the walking group, a decrease in muscle elasticity, an increase in stiffness and muscle strain were found (Figure 1).

\section{DISCUSSION}

Fast-walking exercise was not found to have a positive effect on calf muscle elasticity. We found that the 15-minutes exercise program on the minitrampoline is more effective as a heating technique and has more positive effect on muscle elasticity than the 15-minutes walking exercise.

Studies assessing the effect of exercise on muscle function and stiffness have used different measurement techniques and small cohorts, making it difficult to conclude on the overall efficacy of exercises types on these outcomes. Current data suggest that aerobic exercise training should be used as a primary treatment strategy for improving muscle function $[5,6]$.
Elasticity imaging is a relatively new ultrasoundbased technique for investigating musculoskeletal injury. SE, the most commonly used technique, allows determination of the elastic properties of tissue by applying pressure, which provides a quantitative assessment of muscle tissue as a cheap, practical method [7-9]. Based on current of literature, SE seems to be at least as feasible as ultrasonografi (US) and magnetic rezonance imagination (MRI) for identify clinical muscle-tendon alterations [10].

Green et al. [11] used Magnetic Resonance Elastography (MRE), a noninvasive imaging technique, to assess the time-course of passive elasticity changes in the medial gastrocnemius/soleus muscles before and after a bout of eccentric exercise. Study findings by Crawford et al. [12] suggest that massage loading following eccentric exercise has a greater effect on reducing muscle stiffness.

Passive joint stiffness is an important quantitative measure of flexibility, but is affected by muscle volume and all of the anatomical structures located within and over the joint. Quantifying passive stretching responses of individual muscles by SE, helps the diagnosis of muscle disorders and aids the evaluation of surgical/rehabilitation treatments [13, 14]. The study by Hirata et al [15], showed the significant effectiveness of static stretching on the passive fasciclestiffness. Hirata et al. [16] also examined the muscle stiffness responses of the gastrocnemius/soleus during passive dorsiflexion before and after a static stretching by using US shear 
wave elastography. They indicated that passive muscle stiffness differs among the triceps surae, and that the acute effect of a static stretching is observed only in the stiff muscle. Chino and Takahashi [17], determined muscle elasticity, measured independent of the confounding effects of muscle volume and the other nearby anatomical structures, is associated with passive joint stiffness in the joint position where the muscle is sufficiently lengthened. Chino and Takahashi [18] indicated that variations in the elasticity of other tissues, including medial gastroknemius aponeuroses or the ligaments and joint capsule of the ankle, would be associated with the variations in joint stiffness.

Similar to our study, Yanagisawa et al. [19] assessed the feasibility of SE for measuring exerciseinduced changes in muscle stiffness and to compare the findings of it with those of a tissue stiffness for semi-quantitative assessment. They made participants to perform an arm-curl exercise. They found the strain ratio and the value obtained using the tissue stiffness significantly decreased, after exercise.

In our study, we observed a significant difference in elasticity and stiffness values in the calf muscle groups after 15 minutes on trampoline jumping exercise by SE. When we looked at the strain percentile changes, we also observed a significant decrease in total muscle strains in the trampoline group. We found jumping exercise with minitrampoline as effective as a muscle heating technique. It has been observed in the literature that trampoline studies have resulted an increase in muscle strength, speed, jump, anerobic physical capacity, motor performance, balance and proprioception in long term [20-22]. We measured the positive effect of trampoline on muscle elasticity in the acute phase (in 5 minutes). At 15-minutes fast-walking group a decrease in muscle elasticity, an increase in stiffness and strain were found. Fast-walking exercise was found to be an ineffective heating method compared to trampoline.

SE has to be viewed as an experimental technique without sufficient supporting evidence to be used as a routine examination equivalent to US and MRI in musculoskeletal analysis. The usefulness of SE can be expected to increase rapidly in the musculoskeletal field, as soon as we learn to interpret elastographic artifacts as well as to take advantage of the new information provided by SE [23, 24].

\section{Limitations}

Small sample size and that ultrasound elastography standards have not been established yet in the literature are the limitations of the study.

\section{CONCLUSION}

Elasticity imaging is a relatively new ultrasoundbased technique for investigating musculoskeletal injury. SE, the most commonly used technique, allows determination of the elastic properties of tissue by applying pressure, which provides a quantitative assessment of muscle tissue as a cheap, practical method. Heating with trampoline exercise is an effective, reliable method that generally increases elasticity and reduces stifness in calf muscles. The trampoline-jumping exercise was found to be superior to the fast-walking exercise in acute period.

\section{Conflict of interest}

The authors disclosed no conflict of interest during the preparation or publication of this manuscript.

\section{Financing}

The authors disclosed that they did not receive any grant during conduction or writing of this study.

\section{REFERENCES}

[1] Pedersen M, Fredberg U, Langberg H. Sonoelastography as a diagnostic tool in the assessment of musculoskeletal alterations: a systematic review. Ultraschall Med 2012;33:441-6.

[2] Demirel A, Baykara M, Koca TT, Berk E. Ultrasound elastography-based assessment of the elasticity of the supraspinatus muscle in impingement syndrome: does elastography has any diagnostic value? Clin Rheumatol 2018;37:1661-5.

[3] Xiang X, Yan F, Yang Y, Tang Y, Wang L, Zeng J, et al. Quantitative assessment of healthy skin elasticity: reliability and feasibility of shear wave elastography. Ultrasound Med Biol 2017;43:2:445-52.

[4] Hou SW, Merkle AN, Babb JS, McCabe R, Gyftopoulos S, Adler RS. Shear wave ultrasound elastographic evaluation of the rotator cuff tendon. J Ultrasound Med 2017;36:95-106.

[5] Miklitsch C, Krewer C, Freivogel S. Effects of a predefined mini-trampoline training programme on balance, mobility and activities of daily living after stroke: a randomized controlled pilot study. Clin Rehabil 2013;27:939-47.

[6] Giagazoglou P, Kokaridas D, Sidiropoulou M. Effects of a 
trampoline exercise intervention on motor performance and balance ability of children with intellectual disabilities. Res Dev Disabil 2013;34:2701-7.

[7] Rosskopf AB, Ehrmann C, Buck FM, Gerber C, Flück M, Pfirrmann CW. Quantitative shear-wave US elastographyof the supraspinatus muscle: reliability of the method and relation to tendon integrity and muscle quality. Radiology 2016;278:46574.

[8] Hoskins PR, Martin K, Thrush A. Diagnostic ultrasound : physics and equipment. 2nd ed. Cambridge medicine. Cambridge, UK; New York: Cambridge University Press. xi, 2010: p.263.

[9] Hasegawa H. Kanai H. Ultrasonic methods for measurement of small motion and deformation of biological tissues for assessment of viscoelasticity. Biomedical \& Nanomedical Technologies. Momentum Press: New York, 2015: p.45.

[10] González Hidalgo M, Torres AM, Varona Gómez J. Deformation models : tracking, animation, and applications. Lecture notes in computational vision and biomechanics. Dordrecht; New York: Springer. xiv, 2013:p.297.

[11] Green MA, Sinkus R, Gandevia SC, Herbert RD, Bilston LE. Measuring changes in muscle stiffness after eccentric exercise using elastography. NMR Biomed 2012;25:852-8.

[12] Crawford SK, Haas C, Butterfield TA, Wang Q, Zhang X, Zhao Y, et al. Effects of immediate vs. delayed massage-like loading on skeletal muscle viscoelastic properties following eccentric exercise. Clin Biomech (Bristol, Avon) 2014;29:671-8. [13] Koo TK, Guo JY, Cohen JH, Parker KJ. Quantifying the passive stretching response of human tibialis anterior muscle using shear wave elastography. Clin Biomech (Bristol, Avon) 2014;29:33-9.

[14] Terada S, Miaki H, Uchiyama K, Hayakawa S, Yamazaki T. Effects of isokinetic passive exercise and isometric muscle contraction on passive stiffness. J Phys Ther Sci 2013;25:134752 .

[15] Hirata K, Kanehisa H, Miyamoto N. Acute effect of static stretching on passive stiffness of the human gastrocnemius fascicle measured by ultrasound shear wave elastography. Eur J
Appl Physiol 2017;117:493-9.

[16] Hirata K, Miyamoto-Mikami E, Kanehisa H, Miyamoto N. Muscle-specific acute changes in passive stiffness of human triceps surae after stretching. Eur J Appl Physiol 2016;116:9118.

[17] Chino K, Takahashi H. Measurement of gastrocnemius muscle elasticity by shear wave elastography: association with passive ankle joint stiffness and sex differences. Eur J Appl Physiol 2016;116:823-30.

[18] Chino K, Takahashi H. The association ofmuscleand tendon elasticity with passive joint stiffness: in vivo measurements using ultrasound shear wave elastography. Clin Biomech (Bristol, Avon) 2015;30:1230-5

[19] Yanagisawa O, Niitsu M, Kurihara T, Fukubayashi T. Evaluation of human muscle hardness after dynamic exercise with ultrasound real-time tissue elastography: a feasibility study. Clin Radiol 2011;66:815-9.

[20] Aalizadeh B, Mohammadzadeh H, Khazani A, Dadras A. Effect of a trampoline exercise on the anthropometric measures and motor performance of adolescent students. Int J Prev Med 2016;7:91.

[21] Karakollukçu M, Aslan CS, Paoli A, Bianco A, Sahin FN. Effects of minitrampoline exerciseon male gymnasts' physiological parameters: a pilot study. J Sports Med Phys Fitness 2015;55:730-4.

[22] Giagazoglou P, Kokaridas D, Sidiropoulou M, Patsiaouras A, Karra C, Neofotistou K. Effects of a trampoline exercise intervention on motor performance and balance ability of children with intellectual disabilities. Res Dev Disabil 2013;34:2701-7.

[23] Melo X, Fernhall B, Santos DA, Pinto R, Pimenta NM, Sardinha LB, et al. The acute effect of maximal exercise on central and peripheral arterial stiffness indices and hemodynamics in children and adults. Appl Physiol Nutr Metab 2016;41:266-76.

[24] Klauser AS, Faschingbauer R, Jaschke WR. Is sonoelastography of value in assessing tendons? Semin Musculoskelet Radiol 2010;14:323-33. 